\title{
Cloud monitoring system by Visible-light Fisheye CCD for the Telescope Array
}

\author{
Katsuya Yamazaki ${ }^{*}$, Takayuki Tomida $^{b}$, Ryo Nakamura $^{b}$, Yasunori Saito ${ }^{b}$ for the \\ Telescope Array Collaboration ${ }^{\dagger}$ \\ ${ }^{a}$ Kanagawa University, Yokohama, Kanagawa, Japan \\ ${ }^{b}$ Shinshu University, Nagano, Nagano, Japan \\ E-mail: kyamazaki@kanagawa-u.ac.jp
}

The Telescope Array (TA) is the world-largest cosmic ray detectors in the northern hemisphere, with fluorescence detectors (FDs) and a surface detector (SD) array. We have developed a cloud monitoring system for the TA sky to monitor weather condition during FD observation periods. We use a CCD camera with a fisheye lens to evaluate cloud coverages by identifying stars in the TA sky and comparing with a star catalogue. We will report the hardware of the monitoring system and details of the data analysis.

36th International Cosmic Ray Conference -ICRC2019-

July 24th - August 1st, 2019

Madison, WI, U.S.A.

\footnotetext{
* Speaker.

${ }^{\dagger}$ For collaboration list see PoS(ICRC2019)1177
} 


\section{Introduction}

The Telescope Array (TA) experiment observes Ultra-high energy cosmic rays (UHECRs) with energy more than $10^{18} \mathrm{eV}$ since 2008 in Utah, USA. TA experiment is using two of air shower detection techniques, fluorescence detection technique and particle detection technique to observe UHECRs. We observe secondary particles with particle detector at the ground using scintillation counters. The other method is fluorescence detection technique. We observe fluorescence emission light generated by the interaction between secondary particles and atmospheric molecules using fluorescence detector (FD). TA experiment has three FD stations called Black Rock (BR), Long Ridge (LR) and Middle Drum (MD). Each FD station has 12 or 14 telescopes. Cosmic rays are observed using FD at moonless and cloudless night.

The fluorescence technique is sensitive to atmospheric conditions. Because the fluorescent light generated in the atmosphere reaches the detector while being scattered by the atmosphere. Therefore, TA has several equipments for atmospheric monitoring for calibration. When there is cloud, emitted fluorescence light is scattered by them. In these cases we can't acquire correct data. Therefore it's very important to know the weather, especially cloud existence above the observation site and in the field of view of FDs. TA experiment is using night sky cloud monitoring system with visible CCD camera. This system is installed at 3 sites, BR FD station, LR FD station and Central Laser Facility (CLF) site. The weather is monitored to know the respective weather because each station is at around $20 \mathrm{~km}$ distance. This monitoring system takes picture of night sky every minutes. These images are monitored by operator as reference to judge possibility of operation. It is difficult for person who are not expert of FD operation to judge the weather and to decide continuing observation, because the standard of the weather judgement is depending on observers. We developed the cloud monitoring system to judge the weather by systematic evaluation. In this paper, we report our progress of development.

\section{Cloud Monitoring System}

Cloud monitoring system uses a visible light CCD camera with a fisheye lens installed in a dome housing. The system is easy to set up and can be configured for less than $\$ 5,000$. The visible light CCD camera used is WATEC $120 \mathrm{~N}+$ made by WATEC CO., LTD. The field of view of the fisheye lens is wide as $185^{\circ} \times 154^{\circ} 1^{\prime}$, so it is possible to see the whole sky. The image server uses mmEye-S manufactured by Brains. The generated image size is $720 \times 480$ pixels. It has the resolution and dynamic range that can recognize stars sufficiently in the night sky. This CCD camera exposes for 8 seconds every minute and takes an image every minute. Figure 1 shows overview of the system. In order to operate stable, the system has heater to melt snow, and has fan to remove mist in the dome for winter observation. Another fan is installed to cooling the CCD camera. Figure 2 is inside view of CCD dome housing.

Acquired pictures are transferred to the mmEye-S installed in the building and stored on USB memory. Then, the photo is transferred to other PCs through the network and analyzed for display current weather to FD operator. 


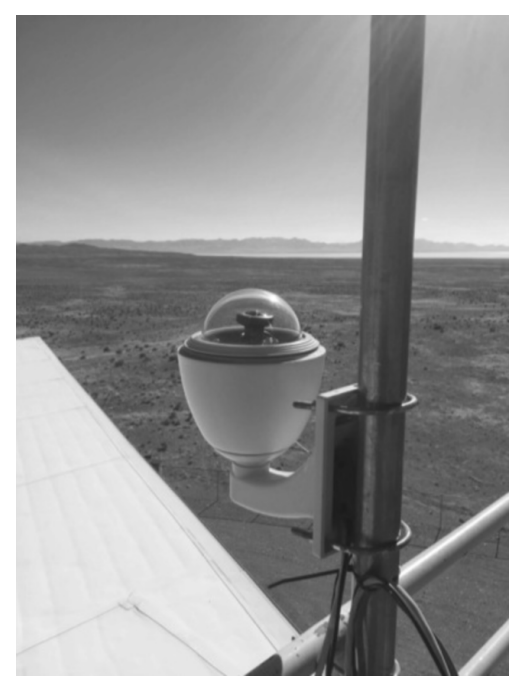

Figure 1: CCD cloud monitoring system overview. The system is installed on a roof of each building.

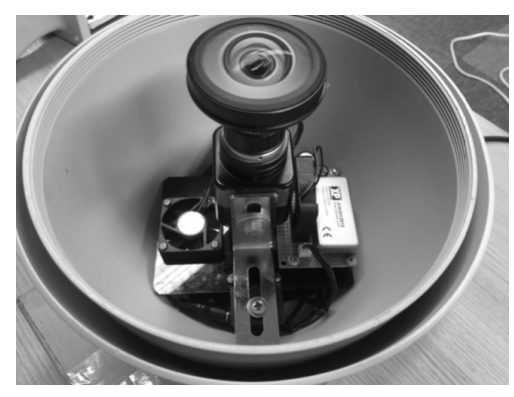

Figure 2: Inside view of CCD dome housing. There are two fans and CCD camera with fisheye lens.

\section{Analysis}

Figure 3 show example of images taken by this system. As shown in Figure 3(a), in a fine-sky image, many stars and the Milky Way are seen, and the whole image looks bright. However, as shown in Figure 3(c), it can be seen that the cloudy image looks dark overall and most stars are invisible. As shown in Figure 3(b), in a partially cloudy image, only a narrow area is visible. It turns out that the weather can be evaluated by detecting stars from these images and counting their numbers. We developed software for detecting bright pixel clusters like stars from images of the night sky.

Firstly, detecting and tagging stars in the pictures using contour detection technique[1]. Bright pixels are separated into three types that stars, noises and dead pixels. Noises caused by town light, air plane and also milky way (can not divide into individual stars). Dead pixel is always bright hot pixel caused by sensor deterioration. Then, we calibrate the field of view of CCD camera by comparing these star positions with astronomical star catalog. We use Smithonian Astrophysical Observatory (SAO) Star Catalog for this purpose. Figure 4 shows star positions in CCD picture coordinate converted from the catalog and Figure 5 shows the result of matching. 


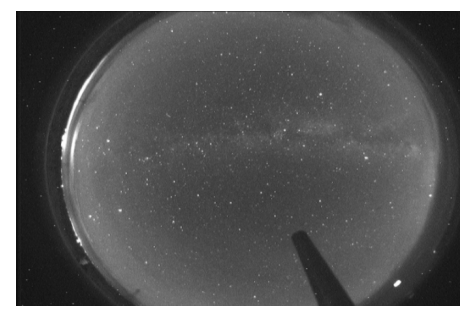

(a) Clear sky

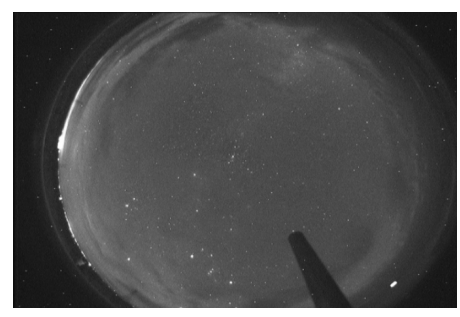

(b) Partly cloudy sky

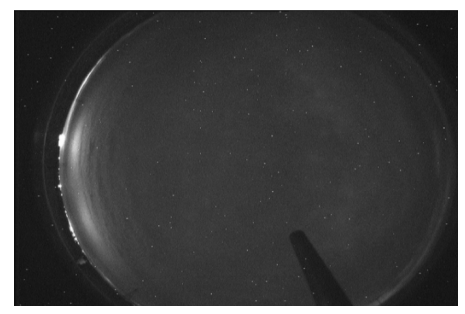

(c) Cloudy sky

Figure 3: Example of picture images. (a) is clear sky image. we can find many stars and milky way in the picture. (b) is partly cloudy sky image. There is cloud in the right area of field of view. (c) is overcast sky image. The sky is overcast and some white spots can be seen in the picture. Most of these white spots are dead pixels and removed in later analysis.

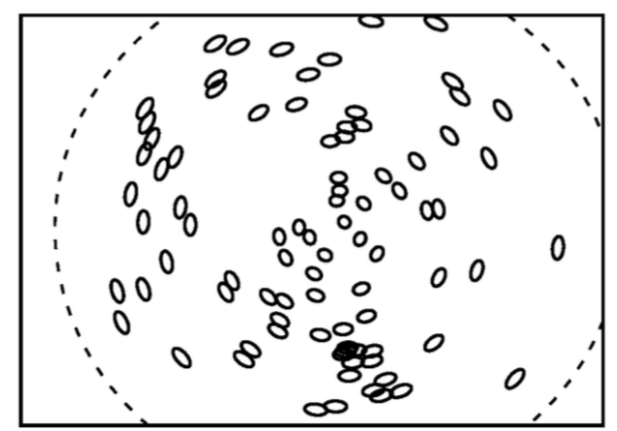

Figure 4: Star positions converted to $\mathrm{CCD}$ picture coordinate from SAO star catalog. Black solid circles are star positions from the catalog. Dashed line indicates the field of view of CCD camera.

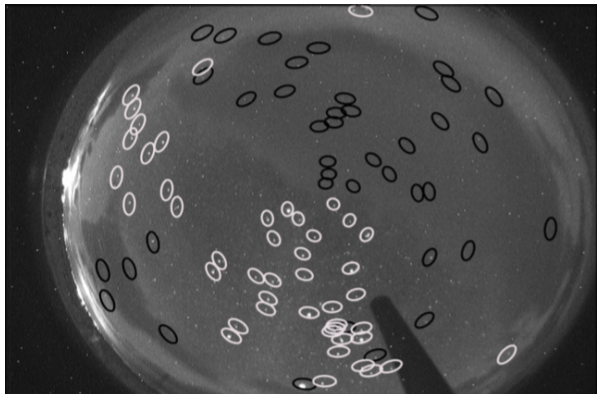

Figure 5: Matched result of CCD picture and the catalog. Gray circles are matched catalog stars. Black circles have no corresponding stars in the CCD picture due to cloud covering.

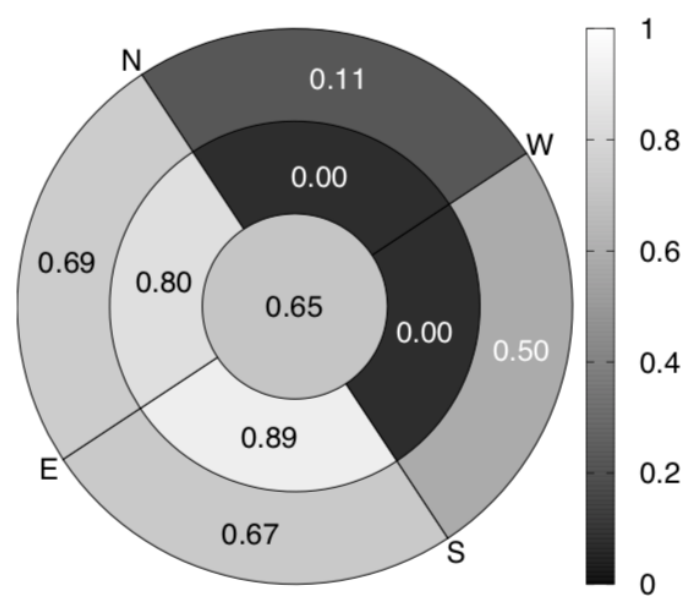

Figure 6: Weather score calculated by Figure 5. The numbers in each area is $R$ of Equation 3.1. 
Finally, we calculate the weather score using ratio of the number stars in the picture by matching with the star catalog. When stars in the picture are matched with star catalog, there is no cloud on that direction. We divide the field of view into nine directions and calculate ratio of matched stars with following equation,

$$
R=\frac{N_{\mathrm{m}}}{N_{\mathrm{e}}}
$$

where $N_{\mathrm{m}}$ is the number of matched stars with the catalog, $N_{\mathrm{e}}$ is the number of expected stars from the catalog and $R$ is ratio of them. Thus, higher $R$ indicates more clear sky in each direction. Figure 6 is result of the calculation for the picture of Figure 5.

We calculate the ratio for each picture, therefore we can get one minute time variation of weather score. Figure 7 shows the time change of the weather score (every 30 degrees in elevation angle). The weather is changing from cloudy to fine. Figure 8 is a picture and weather score taken from each timing shown by the gray dotted frame in Figure 7 (duration 1 to 3). Duration 1 in Figure 7 is cloudy, duration 2 is partly cloudy and duration 3 is clear seeing sky. We can see that the weather is changing with time by time. On the other hand, despite to the clear sky in Figure $8(c)$, weather score indicate slightly low in lower elevation areas. That is caused by atmospheric aerosol contribution. The appearance of the star depends on the elevation angle. Therefore, weather conditions should be set for each elevation angle.

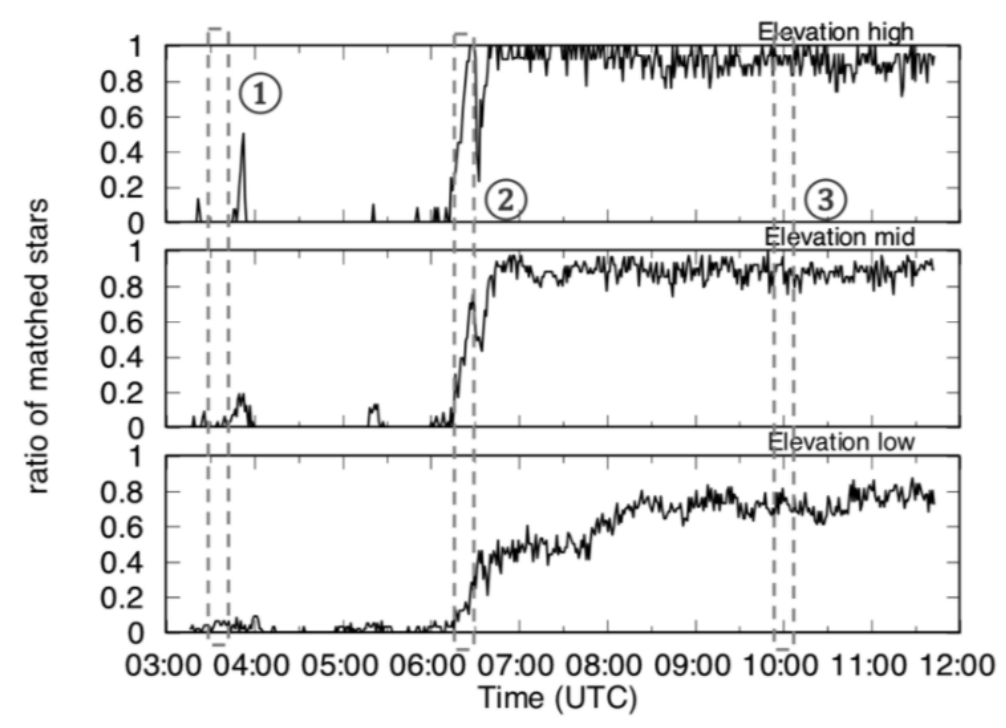

Figure 7: Time variation of the weather score in a day. Three parts of figure are divided by elevation angle region. Top part is high elevation angle $\left(60^{\circ}<\alpha<90^{\circ}\right)$. Middle part is medium elevation angle $\left(30^{\circ}<\alpha<60^{\circ}\right)$. Bottom part is low elevation angle $\left(0^{\circ}<\alpha<30^{\circ}\right)$.

\section{Discussion}

We are developing cloud monitoring system using CCD camera with fisheye lens. By taking night sky pictures with CCD camera every 1 minute, we monitor the weather in detail and with sufficient time resolution. Currently, the threshold of weather decision is tentative value. Next step 


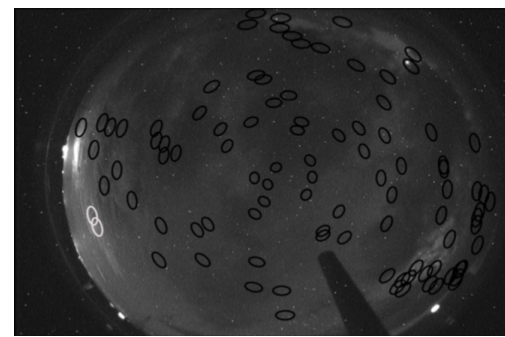

(a) Picture of duration 1.

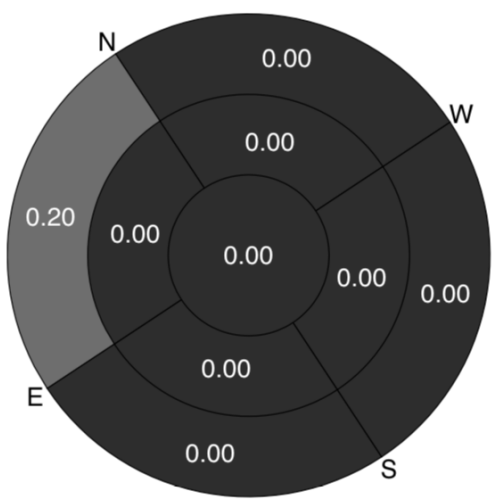

(d) Weather score of duration 1 .

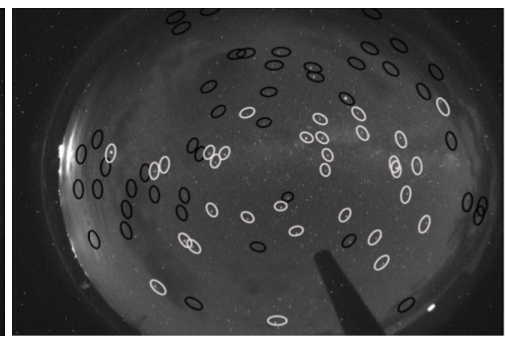

(b) Picture of duration 2.

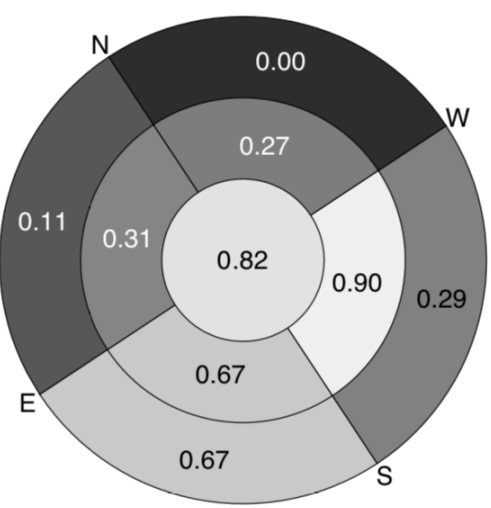

(e) Weather score of duration 2.

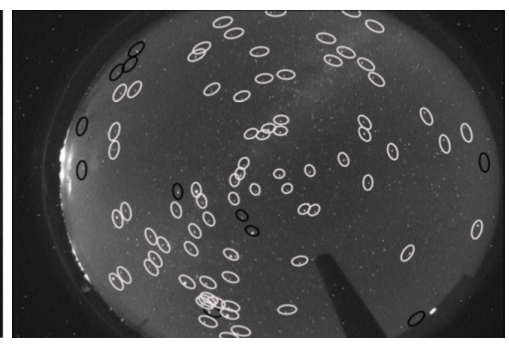

(c) Picture of duration 3 .

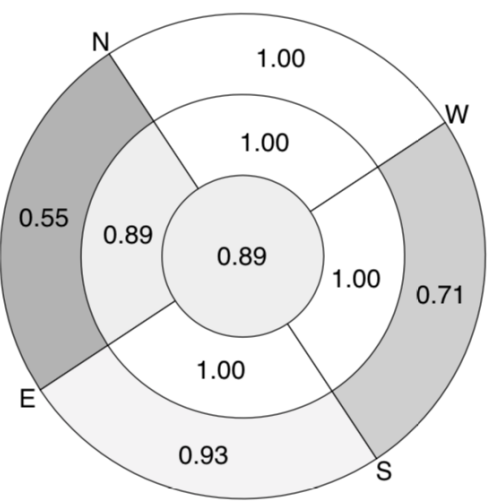

(f) Weather score of duration 3.

Figure 8: Pictures and weather scores at the times indicated by gray dashed box duration 1 to 3 in Figure 7.

of our study is to decide standard value of sky clearness. Our first motivation is to help the FD operation. Therefore, we started providing the real time analysis results to the FD operator with HTML page.

Near future, we will advance the system to decide weather automatically for fully automatic operation of FDs.

\section{References}

[1] T. Tomida et al., Proc. of 35th ICRC, 430, 2017. 